\title{
Speech production planning affects phonological variability: a case study in French liaison
}

\author{
Oriana Kilbourn-Ceron \\ McGill University
}

\section{Introduction}

Connected speech processes have played a major role in shaping theories about phonological organization, and how phonology interacts with other components of the grammar. In particular, processes that can apply across word boundaries, often called "external sandhi," have been important to the development of theories of the syntax-phonology interface (Selkirk, 1974; Kiparsky, 1982; Kaisse, 1985; Nespor \& Vogel, 1986). External sandhi processes are subject to locality restrictions that appear to be syntactic, or correlated with syntactic structure.

But external sandhi processes also seem to be variable above and beyond locality restrictions, in that they are often "optional" even when locality is held constant. As phonological variability has come to the forefront of phonological research programmes, several theories have been and are being developed to understand variable realizations, especially in spontaneous speech. Applying these ideas to external sandhi processes has been part of these developments, since they are indeed consistently variable and by definition apply only in connected speech. One strand of research has focused on the role of probability in predicting the prevalence of reduction processes such as coronal stop deletion and flapping in English, e.g. Gregory et al. (1999) proposing the Probabilistic Reduction Hypothesis (Jurafsky et al., 2001). Relatedly, the Smooth Signal Redundancy Hypothesis ties prosodic modulations to the information density of an utterance, where duration (for example) is increased for more informative (i.e. less predictable) words (Aylett \& Turk, 2004; Turk, 2010). Especially in conjunction with gesturally-based theories of phonology, these approaches show potential in accounting for some patterns of variability in reductive external sandhi processes.

However, an explicit link has not been drawn between external sandhi's two core puzzles of locality and variability. This paper proposes that there is indeed a link between these two properties of external sandhi, through the locality of speech production planning.

1.1 Locality of Production Planning The Locality of Production Planning Hypothesis (PPH) (Wagner, 2012; Tanner et al., 2015; Kilbourn-Ceron et al., 2016) proposes that the constraints of online speech production planning play a role in explaining external sandhi patterns. The core idea is that the choice of pronunciation for a word cannot be affected by the following phonological context if that context is not available at the time the word is being encoded.

According to influential models of speech production (Levelt et al., 1999; Dell \& O’Seaghdha, 1992), planning of connected speech proceeds hierarchically and incrementally. Units like syllables and prosodic words are planned before detailed segmental information, and larger units are planned further in advance than smaller ones(Sternberg et al., 1988; Wheeldon \& Lahiri, 2002; Ferreira \& Swets, 2002). Consequently, at the moment of phonological encoding for a particular word, more details of the higher-level utterance structure are known than lower-level information, e.g. segmental content of upcoming words.

The PPH rests on the idea that the size of planning "chunks" for phonological encoding, being relatively small (Levelt et al., 1999; Wheeldon \& Lahiri, 2002; Wheeldon, 2013), may not always encompass two adjacent words. Hence some words may be phonologically encoded in the absence of information about segments in an upcoming word, preventing interaction between the two words, and therefore blocking external sandhi processes from applying. Furthermore, there is evidence that the size of the planning window

\footnotetext{
* This paper has greatly benefitted from discussions with Michael Wagner, Meghan Clayards, Morgan Sonderegger,
} Jeffrey Lamontagne, James Tanner, and the audience of the Annual Meeting on Phonology 2016.

(C) 2017 Oriana Kilbourn-Ceron

Proceedings of AMP 2016 
is not fixed: It expand or contract depending on many factors, including cognitive load (Wagner et al., 2010), complexity of an upcoming syntactic constituent (Ferreira, 1991), and working memory load (Slevc, 2011). This leads to a potential explanation for different patterns of variability under different speaking conditions.

Kilbourn-Ceron et al. (2016) investigated the PPH prediction that syntactic structure and lexical frequency should influence the probability of flapping coronal stops in English. We tested the effect of syntactic constituency on the likelihood of flapping across a word boundary in a production experiment. Subjects read aloud sentences sentences that included nonce verbs in an embedded clause, and varied whether the following noun was its direct object or the subject of the following clause, i.e. whether the target word was followed by a clause boundary. A logistic regression analysis showed significantly lower likelihood of flapping in the presence of a clause boundary. However, the effect of clause boundary present was gradient, not blocking flapping completely but decreasing the likelihood by about half. The PPH predicts both aspects of the syntax effect found in this experiment: syntax has probabilistic and relatively subtle indirect effect through its influence on the course of speech production planning.

Kilbourn-Ceron et al. (2016) also reports a corpus study testing the effect of lexical frequency of both the target coronal-final word and following vowel-initial word (e.g. cat attack). Many studies have shown that high lexical frequency facilitates word form retrieval (Oldfield \& Wingfield, 1965; Jescheniak \& Levelt, 1994). Accordingly, the PPH predicts that lexical frequency should influence external sandhi in a very specific way: the higher the frequency of word following the target word, the more likely the process should be to apply, since the words are more likely to be encoded within the same planning window. This prediction was tested for flapping using data from the Buckeye Corpus of Conversational Speech, and it was found that indeed there is a statistically significant increase in the likelihood of flapping as the frequency of the word following the target increases. Under the view that flapping is a reductive process due to gestural overlap with adjacent vowels (e.g. Fukaya \& Byrd, 2005), this finding fits in well with the broader research on probabilistic effects on reduction (Bybee \& Scheibman, 1999; Jurafsky et al., 2001; Bell et al., 2003). But unlike, for example, the Probabilistic Reduction Hypothesis of Bell et al. (2003), the predictions of PPH extend also to non-reductive sandhi processes, predicting similar effects of lexical frequency in both cases.

Hence, in the present study, we test the same prediction for a non-reductive process: liaison in French. Since liaison is not a reductive process, only the PPH predicts a similar pattern in the case of liaison. Therefore, the main research question of this paper is whether the lexical frequency of the second word in a potential liaison pair has a positive correlation with the rate of liaison. Such an effect would be evidence that difficulty in retrieving/planning the liaison-triggering word modulates the rate of liaison realization, as predicted by the PPH. If the locality of production planning does indeed play a role in shaping the pattern of external sandhi variability, then it will be crucial to take these effects into account in future empirical studies of large corpora, especially in spontaneous speech.

We address the research question by presenting an analysis of data from the Phonologie du Français Contemporain (PFC) corpus. Section 2 reviews previous research on liaison and its variability, followed by the presentation of the data set used for the present study in Section 3. Section 5 presents the results of the quantitative analysis, and Section 6 discusses how they bear on the PPH and other accounts of variability. Section 7 concludes.

\section{Liaison}

The liaison alternation in French is one in which a subset of words (W1) surfaces with a latent final consonant when the following word (W2) begins with a vowel. For example, the adjective gros 'big' is pronounced [gro] in isolation or before a consonant, but with a final [z] when it modifies a vowel-initial noun as in gros enjeu 'big stake/issue'. There is an extensive literature on liaison, and ongoing debates regarding the lexical affiliation of the liaison consonant (Morin, 2003; Côté, 2005) as well as the apparent morphosyntactic restrictions on the process. The former will not be addressed in detail in this paper as it is not crucial to our point, though the issue is briefly touched on in the discussion (see Côté (2012) for a review).

The locality conditions on liaison have been discussed by many authors, and analyses range from syntactic (e.g. Kaisse, 1985; Pak, 2008) to morphological Steriade (1999); Tranel (1996), to prosodic/phonological (Féry, 2004). Part of what makes liaison's locality conditions a particularly challenging puzzle is that the restrictions vary from "obligatory" to "optional/variable" to "prohibited". This study focuses on two particular syntactic contexts, which are on the spectrum of "variable," as the particular 
interest is how variability might be modulated by lexical frequency.

The first context analyzed is an adjective followed by a noun (Adj-Noun, hereafter), which has been described as obligatory (Delattre, 1955). However, Durand \& Lyche (2008) noted several counterexamples in the PFC data they examined (a subset of the data used for this study).

The second context is between a plural noun and following adjective (PINoun-Adj). The PINoun-Adj context may be less consistent: Between a noun and an adjective, liaison is reportedly impossible, unless the noun is plural, in which case the liaison consonant [z] can be realized. One important difference to note between the PINoun-Adj context and the Adj-Noun context is that post-nominal adjectives are consistently phrased separately from the noun, prosodically. Although liaison can be realized across large boundary, the prosodic phrasing of this context (as opposed to prenominal adjective, which can be phrased with the noun) may lead to overall lower rates of liaison in the context. This a difference which could be interesting to explore from a PPH perspective in future work (see Kilbourn-Ceron et al., 2016, 2017: for PPH-based investigations of syntactic effects). However, in this study, the crucial question is not whether the baseline rates are or even should be the same the two syntactic contexts, but whether they are both similarly modulated by lexical frequency.

This case study differs from the English-based PPH case studies in several ways. Firstly, the process is not reductive, in the sense that the context-dependent variant involves extra articulation. Second, the alternation takes place only at word junctures, in the sense that there is no comparable word-internal alternation. Finally, there are clearly morpho-syntactic restrictions on which words can form a liaison sandhi pair, in contrast with flapping, which applies to all coronal-final words, and is possible across any type of syntactic boundary (Kahn, 1976).

Despite these differences, the PPH predicts a parallel pattern of variability for liaison as we found for flapping in English (Kilbourn-Ceron et al., 2016), since both alternations are dependent on a following context: Higher frequency words are easier to retrieve/plan, so higher frequency should correlate with higher likelihood of both words being planned within the same window, and consequently the realization of the contextual variant. There is evidence from a previous study that probabilities do indeed affect liaison in this way: Côté (2013) found a correlation between liaison rate and the predictability of W2's syntactic category. For a given W1, the rate of liaison was highest for those words for which the category of the following word was most predictable. For example, très 'very' appeared exclusively before adjective/adverbs in their analysis, and its liaison rate was the highest of all the adverbs they examined, at $84 \%$. In contrast, moins 'less' only appeared before adjective/adverbs 53\% of the time, and its liaison rate was only $14 \%$. Although a more detailed syntactic examination is needed, Côté 2013's results are prima facie compatible with PPH predictions: If syntactic predictability facilitates retrieval of $\mathrm{W} 2$, the PPH predicts higher rates of liaison for higher predictability.

\section{Data set}

The source of data for this study was the Phonologie du Français Contemporain corpus (PFC; Durand et al., 2001, 2009), a geographically diverse corpus of read speech and spontaneous conversations. A subset of this corpus is partially annotated for liaison, containing data from 417 speakers recorded in 39 different regions. This was the subcorpus we retrieved via the online search tool ${ }^{1}$. The annotation records 0 for no liaison, 1 for liaison enchainée (forward-linked liaison, the typical case). We restrict our data to observations with either of those annotations, removing cases coded as liaison non-enchainée, uncertain, and 'epenthetic' liaison. This resulted in loss of less than 1\% of the data, leaving 52953 tokens of potential liaison sites.

From this subset were extracted PINoun-Adj pairs ( = 13306) and Adj-Noun pairs ( $\mathrm{n}=2477)$. This was done by retrieving part-of-speech information from the Lexique database (B. et al., 2001:Version 3.81), and matching it orthographically with the PFC data. Lexique is also the source for the lexical frequency information, the main measure of interest in this study. Lexique provides several frequency measures; We chose to use the lemma frequency calculated from movie subtitles, which more closely approximates spoken French. Speech rate was calculated in words per second for each utterance, using the start and endpoints and orthographic transcription provided in the PFC, as it has been noted that liaison may be less frequency in faster speech, which is typically more reduced (Durand \& Lyche, 2008).

1 http://public.projet-pfc.net/liaison/ 


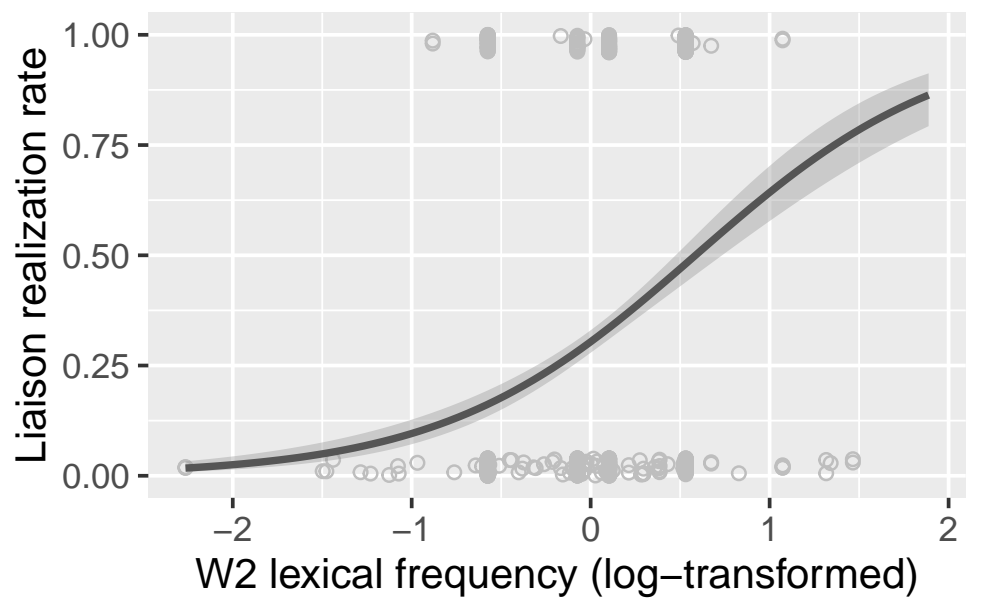

Figure 1: Plural Noun-Adjective contexts: rate of liaison by frequency of W2

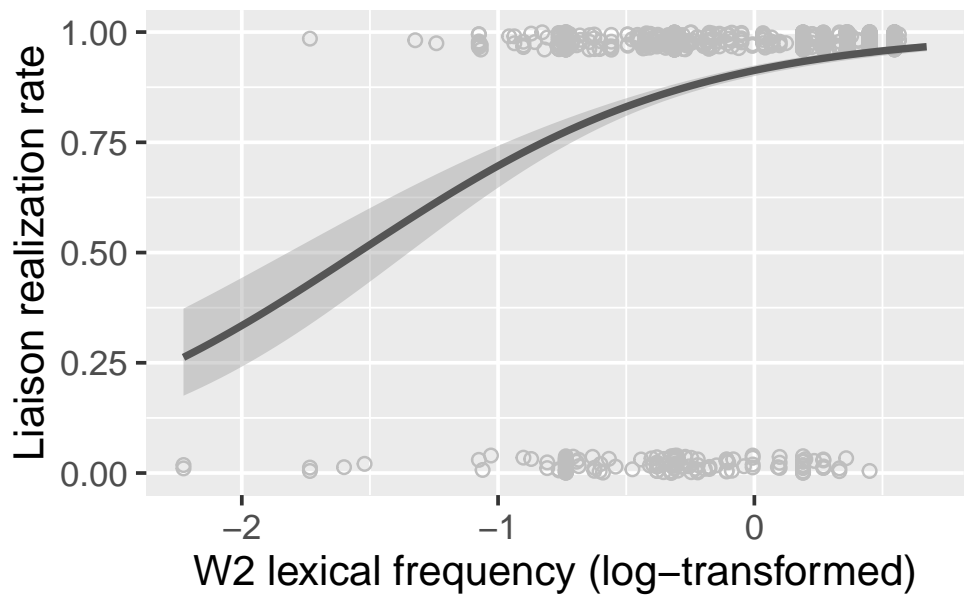

Figure 2: Adjective-Noun contexts: rate of liaison by frequency of W2

Figure 1 shows the correlation between the probability of liaison realization and the frequency of Word 2 (log-transformed) for the PINoun-Adj context, and Figure 2 shows the same for the Adj-Noun context. Both plots suggest a positive correlation, i.e. the likelihood of a liaison consonant being realized increases as the frequency of the following word increases.

\section{Methods}

To verify that the empirical trends were statistically significant after controlling for duration and individual word and speaker differences, a mixed-effects logistic regression was fit to the data. This type of regression predicts the log-odds of a binary outcome, in this case whether or not the the liaison consonant is realized. Using a mixed-effects model allows the inclusion of both fixed effects, which estimate the influence of experimental and control variables, and random effects, which account for variability within groupings of observations, e.g. by-speaker (Gelman \& Hill, 2007; Baayen, 2008).

The dependent variable is coded as 0 (no liaison) or 1 (liaison). The model estimates the log-odds of liaison being realized, so positive effect estimates for the independent variables represent an increase in the predicted likelihood of liaison applying. 
Table 1: Model results: Fixed effects coefficients, standard errors, $z$-scores, and $p$-values for all model predictors applied to the Plural Noun-Adjective data set.

\begin{tabular}{lrrrr}
\hline & Estimate & Std. Error & $\mathrm{z}$ value & $\operatorname{Pr}(>|\mathrm{z}|)$ \\
\hline Intercept & -8.596 & 1.052 & -8.173 & 0.000 \\
W2 Frequency & 1.165 & 0.418 & 2.789 & 0.005 \\
W1 Frequency & -0.335 & 0.806 & -0.415 & 0.678 \\
Speech Rate (Words/Second) & 0.111 & 0.314 & 0.354 & 0.723 \\
W1 Frequency:W2 Frequency & -0.211 & 0.944 & -0.224 & 0.823 \\
\hline
\end{tabular}

Table 2: Model results: Fixed effects coefficients, standard errors, $z$-scores, and $p$-values for all model predictors applied to the Adjective-Noun data set.

\begin{tabular}{lrrrr}
\hline & Estimate & Std. Error & $\mathrm{z}$ value & $\operatorname{Pr}(>|\mathrm{z}|)$ \\
\hline Intercept & -6.014 & 1.753 & -3.431 & 0.001 \\
W1 Frequency & 1.620 & 0.901 & 1.798 & 0.072 \\
W2 Frequency & 0.441 & 0.181 & 2.431 & 0.015 \\
Speech Rate (Words/Second) & 0.158 & 0.152 & 1.044 & 0.297 \\
W1 Frequency:W2 Frequency & 1.144 & 0.438 & 2.614 & 0.009 \\
\hline
\end{tabular}

In terms of fixed effects, lexical frequency for $\mathrm{W} 1$ and $\mathrm{W} 2$ were included as continuous variables, $\log$-transformed to bring the distribution closer to normality, and standardized (centred and divided by two standard deviations Gelman \& Hill, 2007). Speech rate in words-per-second was included as a control, also standardized. The model also includes an interaction term between W1 and W2 frequency

Random effect structure included by-speaker and by-liaison word intercepts, which allow the model to take into account baseline differences between speakers and between words. The model also includes byspeaker random slopes for liaison word frequency and next word frequency, which increases the accuracy of p-values and coefficient estimates for the corresponding fixed effects terms and helps ensure their generalizability across speakers (Barr et al., 2013).

\section{Results}

The overall rate of devoicing for the PINoun-Adj context was $23.21 \%(\mathrm{n}=1581)$, and $58.54 \%$ for the Adj-Noun context $(n=2477)$.

The results of the statistical model show that the empirical correlations shown in Figures 1 and 2 between the likelihood of liaison and the frequency of W2 are statistically reliable. The fixed-effects estimates for the PINoun-Adj context, shown in Table 1, show that W2 frequency is the only statistically significant predictor for this context $(\beta=1.165, p=0.005)$. This estimate means that, with other predictors held at mean values, the odds of realizing the liaison consonant increase about 3.2 fold for every increase in one standard deviation of W2 frequency.

In the Adj-Noun model, shown in Table 2, the W2 frequency effect was also significant $(\beta=0.441$, $p=0.015$ ). This represents an increase in the odds of devoicing by about 1.6 for every increase in one SD of W2 frequency, a slightly bigger effect but still comparable to that found for PINoun-Adj. In addition, the interaction between W1 and W2 frequency was significant for Adj-Noun $(\beta=1.144, p=0.009)$. Intuitively, this means that as W1 increases in frequency, the positive effect of W2 frequency increases beyond what would be expected from simply adding the effects of W1 and W2 frequency. That is, the more frequent W1 is, the more an increase in W2's frequency increases the likelihood of liaison. No other effects reached statistical significance. 


\section{Discussion}

The results of statistical modeling confirm that the frequency of W2 in a liaison sandhi pair has a significant positive effect on the realization of liaison in both the Adj-Noun and PINoun-Adj contexts. Given that lexical frequency has a facilitatory effect on word form retrieval (Oldfield \& Wingfield, 1965; Jescheniak \& Levelt, 1994), the W2 frequency effect is consistent with the PPH predcition that liaison should be more likely when W2 is easier to plan. Again, this prediction extends to all external sandhi processes in which the the triggering environment is found across a following word boundary, and there is supporting evidence for this pattern from previous studies on English coronal stop realizations (Tanner et al., 2015; Kilbourn-Ceron et al., 2016). The finding of a parallel effect of lexical frequency for both general reductive processes like coronal stop deletion and flapping in English, and for a non-reductive process like liaison in French is, we argue, uniquely predicted by the PPH. To show this, we turn to a discussion of possible alternative accounts of frequency effects.

Probability-based accounts Kilbourn-Ceron et al. (2016) discusses how probability-based accounts of reduction like Jurafsky et al. (2001) make overlapping predictions with the PPH. Kilbourn-Ceron et al. (2016)'s finding that W2 frequency has a positive correlation with the likelihood of flapping could alternatively be interpreted as a reduction due to higher predictability of the following word and consequently a reduced flap realization of the word-final coronal stop. However, given that liaison involves extra articulation relative to its pronunciation in other environments, the positive frequency effect cannot be straightforwardly derived from this type of account. While we do not discount the possibility of probabilitybased reduction effects, we submit that finding a W2 frequency effect in liaison points to a need to acknowledge the role that general speech production planning constraints play in shaping phonological variability. Indeed, the PPH proposes a specific cognitive mechanism that could be at the source of some probability-based reduction effects.

One phonological difference to note between liaison and flapping is that the articulation proper of the liaison consonant does necessarily have to be tightly coordinated with the gestures of the following vowel. It is possible to pronounce the liaison variant with a pause afterwards, unlike a flap, which requires detailed coordination with the following vowel it is being released into. We hypothesize that this may lead to slightly less sensitivity for liaison than for flapping, since less detailed planning is necessary for the contextual variant. For the purposes of isolating and teasing out evidence of production planning effects, the crucial difference between liaison and flapping is the non-reductive aspect. Probability-based theories of variable pronunciations make overlapping predictions with the PPH for reductive processes like flapping, but make no or opposite predictions for non-reductive processes. If it is the case that lexical frequency of the liaisontriggering word modulates variability in the same way as it did for flapping, this would support the idea that online speech production planning effects play a role in shaping phonological variability.

Information-theoretic approaches A related stream of research has tied phonetic and phonological variation to effective message transmission (Aylett \& Turk, 2004; Currie Hall et al., 2016; Cohen Priva, 2017). Under this view, phonetic and phonological patterns reflect the pressure to encode a uniform amount of information throughout the utterance. For example, more frequent words are temporally compressed or articulatorily reduce because they contribute less information to the listener. Currie Hall et al. (2016) mention liaison in particular: they propose that liaison is avoided in less predictable contexts because the realization of the liaison consonant masks the boundary between W1 and W2, creating difficulty for retrieval. This is compatible with the results presented above, and compatible with the PPH in general: The PPH could be considered a specific mechanism by which some of these effects come about. However, the predictions of the PPH differ from alternative approaches in that they are more constrained. Under a message oriented approach like Currie Hall et al. (2016), it is possible to imagine an opposite prediction for liaison: The realization of liaison signals the fact that the following word starts with a vowel, so it should be advantageous to realize liaison in less predictable contexts. For example, in the phrase mon peti[t] ami 'my little friend', the realization of liaison gives an early cue that the head noun ami starts with a vowel, which could facilitate retrieval. ${ }^{2}$ This type of prediction is incompatible with the PPH, as it would imply that a process that makes

\footnotetext{
2 A possible counter-argument is that petit with liaison is homophonous to the feminine form petite, which could cause liaison to introduce further ambiguity. However, the determiner in the phrase has already marked the masculine gender
} 
reference to information in the upcoming word applies more when the word is less likely to be planning within the same window. Such a pattern would falsify the PPH, but both this pattern and its opposite could be accommodated under, for example, Currie Hall et al. (2016).

Lexical affiliation of liaison consonant In this study we have made the simplifying assumption that the liaison consonant is final in $\mathrm{W} 1-$ now, we briefly address an alternative view. It has been proposed in previous work that the liaison consonant is in fact affiliated with both W1 and W2, both lexically and in terms of surface syllabification (L'Esperance, 2015; Smolensky \& Goldrick, 2016). Smolensky \& Goldrick (2016) propose that both W1 and W2 are associated with a final and initial consonant respectively, and both consonants carrying some 'activation weight' that, only when combined, result in the pronunciation of the liaison consonant. This view seems compatible with the PPH account of variability that we suggest here-if W1 and W2 must both be encoded within the same window in order for the liaison consonant to be realized.

Difference by syntactic context In the results of the statistical model, the interaction between W1 and W2 frequency was only significant for the Adj-Noun construction. While the main goal of this paper was to investigate the pattern of W2 frequency effects, we offer some thoughts on why the interaction may show different patterns. It has been argued that the difference between prenominal and postnominal adjectives in French is not merely a difference in linearization, but also syntactic structure. Bouchard (1998) argues that there is less structure between a prenominal adjective and the head noun than between a noun and postnominal adjective. If this is the case, under the PPH we might expect the Adj-Noun to be more likely to be encoded within the same planning window than a noun and postnominal adjective, leading to more complex frequency effects on liaison realization. In future work, it would be interesting to more carefully probe the interaction between syntactic structures and frequency effects.

\section{Conclusion}

This paper has shown that the realization of a variable liaison consonant in two different syntactic contexts is dependent on the lexical frequency of the second word in the sandhi pair. This lends support to the idea that locality of production planning plays a role in phonological variability above and beyond temporal and gestural reduction effects. This is because liaison, a non-reductive sandhi process, shows the same pattern of variability as reductive processes like flapping (Kilbourn-Ceron et al., 2016, 2017), a prediction that is crucially not derivable from hypotheses that based on probabilistic reduction, signal redundancy, or information modulation. The PPH, on the other hand, explains these parallel patterns by reference to the size of the planning window for phonological encoding, predicting that variants that depend on following context, like liaison and flapping, can only be realized if the following context is sufficiently planned. Factors like lexical frequency (among many others), which delay retrieval and encoding, modulate the availabilility of the following context and therefore reduce the probability of planning the contextually "appropriate"variant. Future work could test the effect on external sandhi of a range of other factors that have been shown to modulate difficulty of speech production planning, such as syntactic complexity of an upcoming constituent (Ferreira, 1991), number of words in the utterance(Wheeldon \& Lahiri, 2002; Wheeldon, 2013), and even codability of the noun in an upcoming constituent (Lee et al., 2013).

We suggest that accounting for production planning effects is important to understanding which parts of pronunciation variability are part of a speaker's knowledge of their language, and which parts are a consequence of general cognitive processes. Evidently, online speech production planning constraints may not be the only source of non-linguistic phonological variability, but developing an explicit hypothesis about these effects in particular could be part of a more complete understanding of human language output.

of the upcoming phrase. And interestingly, in Laurentian French, the distinction between liaison- and fixed-consonant forms (i.e. masculine and feminine in this case) is marked by laxing of the final vowel in the fixed-consonant form, further mitigating ambiguity. One remaining ambiguity could be with a W2 that begins with the same consonant, as in petit tamis. Future work could investigate whether liaison is disfavoured in pairs that are ambiguous in this way, versus pairs in which no lexical item could correspond to W2, e.g. petit ours-petit *tours. 


\section{References}

Aylett, Matthew \& Alice Turk (2004). The smooth signal redundancy hypothesis: A functional explanation for relationships between redundancy, prosodic prominence, and duration in spontaneous speech. Language and speech 47:1, 31-56.

B., New, C. Pallier, L. Ferrand \& R. Matos (2001). Une base de données lexicales du français contemporain sur internet: Lexique. L’Année Psychologique 101, 447-462.

Baayen, R.H. (2008). Analyzing linguistic data. Cambridge University Press, Cambridge.

Barr, Dale J, Roger Levy, Christoph Scheepers \& Harry J Tily (2013). Random effects structure for confirmatory hypothesis testing: Keep it maximal. Journal of memory and language 68:3, 255-278.

Bell, Alan, Daniel Jurafsky, Eric Fosler-Lussier, Cynthia Girand, Michelle Gregory \& Daniel Gildea (2003). Effects of disfluencies, predictability, and utterance position on word form variation in english conversation. The Journal of the Acoustical Society of America 113:2, 1001-1024.

Bouchard, Denis (1998). The distribution and interpretation of adjectives in french: A consequence of bare phrase structure. Probus 10:2, 139-183.

Bybee, Joan \& Joanne Scheibman (1999). The effect of usage on degrees of constituency: the reduction of don't in english. Linguistics 37:4, 575-596.

Cohen Priva, Uriel (2017). Not so fast: Fast speech correlates with lower lexical and structural information. Cognition 160, 27-34.

Côté, Marie-Hélène (2005). Le statut lexical des consonnes de liaison. Langages 2, 66-78.

Côté, Marie-Hélène (2012). French liaison. van Oostendorp, Marc, C Ewen, E. Hume \& K. Rice (eds.), Companion to phonology, Wiley-Blackwell.

Côté, Marie-Hélène (2013). Understanding cohesion in french liaison. Language Sciences 39, 156-166.

Currie Hall, Kathleen, Elizabeth Hume, T. Florian Jaeger \& Andrew Wedel (2016). The message shapes phonology. Ms. Univ. of British Columbia, Univ. of Canterbury, Univ. of Rochester, Univ. of Arizona.

Delattre, Pierre (1955). Les facteurs de la liaison facultative en français. French review 42-49.

Dell, G.S. \& P.G. O’Seaghdha (1992). Stages of lexical access in language production. Cognition 42:1-3, $287-314$.

Durand, Jacques \& Chantal Lyche (2008). French liaison in the light of corpus data. Journal of French Language Studies 18:01, 33-66.

Durand, Jacques, Bernard Laks \& Chantal Lyche (2001). La phonologie du français contemporain: usages, variétés et structures.

Durand, Jacques, Bernard Laks \& Chantal Lyche (2009). Le projet pfc (phonologie du français contemporain): une source de données primaires structurées. Phonologie, variation et accents du français 19-61.

Ferreira, F. (1991). Effects of length and syntactic complexity on initiation times for prepared utterances. Journal of Memory and Language 30:2, 210-233.

Ferreira, Fernanda \& Benjamin Swets (2002). How incremental is language production? evidence from the production of utterances requiring the computation of arithmetic sums. Journal of Memory and Language 46, 57-84.

Féry, Caroline (2004). Gradient prosodic correlates of phrasing in french. Nouveaux départs en phonologie $161-182$.

Fukaya, Teruhiko \& Dani Byrd (2005). An articulatory examination of word-final flapping at phrase edges and interiors. Journal of the international phonetic association 35:01, 45-58.

Gelman, Andrew \& Jennifer Hill (2007). Data analysis using regression and multilevel/hierarchical models. Cambridge University Press, Cambridge.

Gregory, Michelle L, William D Raymond, Alan Bell, Eric Fosler-Lussier \& Daniel Jurafsky (1999). The effects of collocational strength and contextual predictability in lexical production. Chicago Linguistic Society, vol. 35, 151-166.

Jescheniak, Jörg D \& Willem JM Levelt (1994). Word frequency effects in speech production: Retrieval of syntactic information and of phonological form. Journal of Experimental Psychology: Learning, Memory, and Cognition 20:4, p. 824.

Jurafsky, Daniel, Alan Bell, Michelle Gregory \& William D Raymond (2001). Probabilistic relations between words: Evidence from reduction in lexical production. Typological studies in language 45, 229-254.

Kahn, Daniel (1976). Syllable-based generalizations in English phonology. Ph.D. thesis, Massachusetts Institute of Technology.

Kaisse, Ellen M (1985). Connected speech: the interaction of syntax and phonology. Academic Press. 
Kilbourn-Ceron, Oriana, Michael Wagner \& Meghan Clayards (2016). The effect of production planning locality on external sandhi: a study in /t/. The Proceedings of the 52nd Meeting of the Chicago Linguistics Society, lingbuzz/003119.

Kilbourn-Ceron, Oriana, Michael Wagner \& Meghan Clayards (2017). External sandhi and the locality of production planning: The case of flapping. Ms., McGill University. Submitted.

Kiparsky, Paul (1982). Lexical phonology and morphology. Linguistics in the morning calm .

Lee, Eun-Kyung, Sarah Brown-Schmidt \& Duane G Watson (2013). Ways of looking ahead: Hierarchical planning in language production. Cognition 129:3, 544-562.

L'Esperance, Marie-Josee (2015). The Phonetics And Phonology Of Liaison Consonants In Montreal French. Ph.D. thesis, Cornell University.

Levelt, Willem JM, Ardi Roelofs \& Antje S Meyer (1999). A theory of lexical access in speech production. Behavioral and brain sciences 22:01, 1-38.

Morin, Yves-Charles (2003). Remarks on prenominal liaison consonants in french. Living on the Edge 28, 385-400.

Nespor, Marina \& Irene Vogel (1986). Prosodic phonology. Walter de Gruyter.

Oldfield, Richard C \& Arthur Wingfield (1965). Response latencies in naming objects. Quarterly Journal of Experimental Psychology 17:4, 273-281.

Pak, Marjorie (2008). The postsyntactic derivation and its phonological reflexes. Ph.D. thesis, University of Pennsylvania.

Selkirk, Elisabeth (1974). French liaison and the x notation. Linguistic Inquiry 573-590.

Slevc, L Robert (2011). Saying what's on your mind: working memory effects on sentence production. Journal of experimental psychology: Learning, memory, and cognition 37:6, p. 1503.

Smolensky, Paul \& Matthew Goldrick (2016). Gradient symbolic representations in grammar: The case of French liaison. Ms. Johns Hopkins University and Northwestern University.

Steriade, Donca (1999). Lexical conservatism in french adjectival liaison. Authier, M., B. Bullock \& L. Reed (eds.), Proceedings of the 25th Linguistic Colloquium on Romance Languages, John Benjamins, 243270.

Sternberg, S., RL Knoll, S. Monsell \& CE Wright (1988). Motor programs and hierarchical organization in the control of rapid speech. Phonetica 45:2-4, 175-197.

Tanner, James, Morgan Sonderegger \& Michael Wagner (2015). Production planning and coronal stop deletion in spontaneous speech. Proceedings of the 18th International Congress of Phonetic Sciences.

Tranel, Bernard (1996). French liaison and elision revisited: A unified account within optimality theory. Aspects of Romance linguistics 433-455.

Turk, Alice (2010). Does prosodic constituency signal relative predictability? a smooth signal redundancy hypothesis. Laboratory phonology 1:2, 227-262.

Wagner, Michael (2012). Locality in phonology and production planning. Proceedings of Phonology in the 21 Century: Papers in Honour of Glyne Piggott. McGill Working Papers.

Wagner, Valentin, Jörg D Jescheniak \& Herbert Schriefers (2010). On the flexibility of grammatical advance planning during sentence production: effects of cognitive load on multiple lexical access. Journal of Experimental Psychology: Learning, Memory, and Cognition 36:2, p. 423.

Wheeldon, LINDA (2013). Producing spoken sentences: The scope of incremental planning. Perrier, P. \& P. Lang Verlag (eds.), Cognitive and physical models of speech production, speech perception, and production-perception integration.

Wheeldon, L.R. \& A. Lahiri (2002). The minimal unit of phonological encoding: prosodic or lexical word. Cognition 85:2, B31-B41. 